\title{
The Prevalence of Bacterial Pathogens
} associated with Urinary Tract Infection (UTI)
among Patients attending KAM Medical
Consult Clinic in Uganda.

\author{
Gerald Turyatungaa, \\ a Faculty of Health Sciences, Uganda Martyrs University
}

Abstract

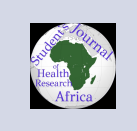

Background: ${ }^{a}$

A urinary tract infection (UTIS) is a common type of infection caused by bacteria that travel up the urethra to the bladder. Globally, it is reported that 150 million people are diagnosed with a UTI annually, costing the world economy over 6 million US dollars in treatment and work loss. Studies conducted in the in-patients pediatric ward of Muhimbili Hospital in Tanzania and Mulago National Referral Hospital in Uganda between five and ten years reported the prevalence of UTI to be $16.8 \%$ and $14.6 \%$ respectively.

On average at Kam Medical Consult Clinic, patients who turn up in the laboratory are about $20 \%$ and $50 \%$ of patients present with recurring UTIs clinically. Therefore, there is a need to establish and investigate the prevalence of bacterial pathogens associated with UTI and multiple pathogens that are not known.

Methodology:

The cross-sectional study was carried out at Kam medical consult clinic (KMCC) located in Kafeero zone Mulago II Kawempe division. The study population were adults and children presenting with symptoms of urinary tract infection. 120 Midstream urine Samples were collected from patients presenting clinical signs of UTI. Diagnosis was done through macroscopy dry chemistry, microscopy, and culture.

\section{Results}

Among the sampled population, $33 \%$ of males had urinary tract infection while $67 \%$ of females had urinary tract infection. 88.5\% were outpatients while $11.5 \%$ were in-patients The overall prevalence of UTI among patients was $63.3 \%$ with children aged (1-10) having a prevalence of $5.8 \%$.

\section{Conclusion and recommendation:}

E.coli is an etiological agent causing UTIs in male and female patients presenting UTI at Kam Medical Consult Clinic. Other etiological agents included Klebsiella, Coliform, Enterococcus species, staphylococcus aureus, pseudomonas species, and candida species. There is a need to monitor the profile of etiological bacteria of UTI through culture and sensitivity regularly.

\footnotetext{
${ }^{a}$ date submitted: 11th/02/2021 date accepted: 8th/04/2021 email: turyatungagerald2014@gmail.com
}

\section{Background}

A urinary tract infection (UTIS) is a common type of infection caused by bacteria that travel up the urethra to the bladder (CDC, 2015). Globally, it is reported that 150 million people are diagnosed with a UTI annually, costing the world economy 
over 6 million US dollars in treatment and work loss (Parveen et al., 2011). Urinary tract infections are among the most frequent bacterial infection worldwide (Odoki et al., 2019). The study in Nigeria reported a prevalence of UTI of $14.2 \%$ (Aiyegero et al., 2007). In Kenya, Hannah et al., (2011) reported the prevalence of UTI to be $18 \%$. Furthermore, other studies conducted in the in-patients pediatric ward of Muhimbili Hospital in Tanzania and Mulago National Referral Hospital in Uganda between five and ten years reported the prevalence of UTI to be $16.8 \%$ and $14.6 \%$ respectively (Whalley et al., 2015). Risk factors for Urinary tract infection (UTI) tend to increase the rate of infection in women due to having sex frequently or having a new sex partner and using a spermicide for birth control (Rahimkhani et al., 2008). Women report to be more susceptible to urinary tract infections because their urethra is shorter(Minardi et al., 2011). Frequent sexual intercourse tends to increase a woman's risk of developing UTIs (Coyne et al., 2012). Furthermore, the use of contraceptive spermicides and diaphragm contribute to risk factors of acquiring infection and age also has been associated with increased risk of UTI. In women in the menopause stage also reported decreasing in estrogen thins the lining of the urinary tract, which increases susceptibility to bacterial infections (Mshana et al., 2014).

Urinary tract infection (UTI) is mainly caused by gram-negative organisms which include Escherichia coli $60-70 \%$, Klebsiella species 10\%, Proteus species 5-10\%, and Pseudomonas aeruginosa 2-5\% (Cheesbrough 2000). However, other gram-positive bacteria associated with UTI include Staphylococcus aureus, Enterococcus faecalis, and Staphylococcus saprophyticus which accounts for the prevalence of $4.6 \%, 3 \%, 1.5 \%$ respectively (Gupta et al., 2001). Furthermore, Stamm et al., (2001) reported that Escherichia coli is the most common pathogen that is associated with UTI in Nigeria and it accounts for $47.6 \%$ and Pseudomonas aeruginosa is the second most common pathogen in Nigeria with 9.2\% ( Wanyama et al., 2003).

The prevalence of UTI (Urinary Tract Infection) globally is on increase at $25 \%$ (Gupta et al., 2010). $50-60 \%$ of adult women experience UTI during their lifetime (Kayima et al., 1996). In young women, 0.5 episodes of acute cystitis per person per year occur and this incidence decreases width age (Moges et al., 2002). Approximately 3.5\% per 10,000 persons are estimated to be infected in England and wales whereas in Italy $2.4 \%$ of a cohort of 450,000 people received a diagnosis of acute cystitis (Gallali et al., 2006). In Uganda, the prevalence of UTI is estimated to be $13.3 \%$ regionally (Byamugisa et al., 2010).

On average at Kam Medical Consult Clinic, patients who turn up in the laboratory are about 20\% and $50 \%$ of patients present with recurring UTIS clinically. The urinary tract is a common bacterial infection affecting men and women. According to standard operating procedures from the Ministry of Health and WHO (2003) recommend culture and other routine method like urinalysis and Gram staining technique to identify bacterial urinary tract infection. Therefore, there is a need to establish and investigate the prevalence of bacterial pathogens associated with UTI and multiple pathogens that are not known. Therefore, it is important to establish specific ways of diagnosing UTI and determine involved bacteria. This will be achieved through laboratory diagnosis like gram staining technique and culture methods to detect microbial infection.

\section{Methodology}

\section{Materials and methods.}

\section{Study area.}

This study was carried at Kam medical consult clinic (KMCC) located in Kafeero zone Mulago II Kawempe division,

\section{Study Population}

The study population were consists of children patients between 1-17 years and adults aged between 18-90 years presenting with symptoms of urinary tract infection attending Kam Medical Consult Clinic

\section{Study Design.}

This is cross-sectional study was done on urine sample at Kam medical consult clinic conducting for 4 months (March to July) 2016. Samples were collected from patients presenting clinical signs of UTI. All adult and children patients reporting to a medical clinic with signs and symptoms of UTI were enrolled and tested by macroscopy dry chemistry, microscopy, and culture. This study population also included pregnant women attending KAM Medical Consult Centre (KMCC).

\section{Sample size.}

The sample size was determined using the Kish and lisle formula (1965) 
$\mathrm{N}=\frac{z^{2} p(1-p)}{d^{2}}$

Where: $z=$ Z score for $95 \%$ confidence interval = 1.96, $p=$ prevalence, $d=$ tolerable error $=5 \%$.

$n=\frac{z^{2} p(1-p)}{d^{2}}$ Where $\mathrm{z}=10, \mathrm{p}=6, \mathrm{~d}=5 \%$.

$n=\frac{10^{2} 6(1-6)}{5^{2}}$

$=120$. Therefore, 120 samples will be analyzed

The prevalence of $10 \%$ was used according to the study by Akinloye et al., (2006).

\section{Inclusion criteria.}

The study included all children of the age 1' to17 years and adults aged 18 to 90 years patients that turn up in the laboratory.

Children and adults without request form but present symptoms of urinary tract infection.

Able to provide a written informed consent form for the adults.

Children whose parents were willing to give informed consent on their behalf were inlcuded in the study.

\section{Exclusion criteria.}

The study did not include children and adults that have been on antibiotics for the previous two weeks. Also, children and adults without urinary tract infection symptoms were excluded.

\section{Sample collection (Urine collection).}

Midstream urine was collected on the same day of enrollment using the sterile container. Sample containers were properly labeled the patients' identity, sex, and age, label the container with patients name, date of birth, and the date, wash your hands, start to urinate, but don't collect the first part of urine that comes out, collect a sample of urine "midstream" in a sterile screw-top container, screw the lid of the container shut, wash your hands thoroughly and immediately Sample would be sent to the laboratory for analysis on the same day.

\section{Sample analysis for UTI.}

Pipetted $3 \mathrm{ml}$ volume of urine using sterile pasture pipette into the transparent tube to observe the appearance of urine and urine was a span at $2000 \mathrm{~g}$ for 5 minutes, the supernatant was poured off and sediment is mixed, $50 \mathrm{ul}$ of sediment will be placed at the Centre of a glass slide, and a coverslip is placed on a slide, wet preparation of the sediment would be examined under the light microscope using $\times 10$ objective $\times 40$ objective lens to look for Epithelial cells, urinary crystals, red cells, yeast cells and the number of leukocytes per microscopic field was recorded. After inoculating the fresh urine sample on culture plates for urine cul- ture, urine microscopy would be then carried out on the rest of the sample. Urinalysis using urine dipstick was used following manufacturer's instructions as follow; look at the appearance of a urine sample for turbidity. Dipstick into the urine up to the test area for two seconds. Draw the edges of the strip along the brim of the vessel to excess urine. Compare the color of the reagent pads after exactly 60 seconds with the color chart on the vial label under good light. Laboratory results will be reported semi-quantitatively.

\section{Microbiological culture method.}

The cysteine lactose electrolyte deficient (CLED), Blood Agar, and MacConkey (Mac) were used as selective media for isolation. Agar was prepared following the manufacturer's instructions and sterilized. The Media was prepared according to the manufacturer's instructions and $0.001 \mathrm{ml}$ of sample inoculated onto media using a platinum wire loop. Plates were incubated for 24 hours at $37^{\circ} \mathrm{C}$. Inoculate using $10 \mathrm{ul} / 0.01 \mathrm{ml}$ (x100) loop onto selective/non selective media by streaking methods, such as CLED, BAP, and MAC. Dip calibrated loop into well-mixed urine and quickly make a single streak down the middle of the BAP with the loop containing urine. Streak back and forth across the plate perpendicular to the original inoculum and this creates a "lawn". With the same calibrated loop, do the same with the MAC plate. The number of pure colony-forming units was multiplied by 1000 to determine the number of microorganisms per milliliter in the original specimen urine. A diagnosis of UTI was considered when there is at least 105 colony-forming units (CFU)/ml of urine (WHO 2003). High colony counts with more than one species of bacteria were considered as contamination. Therefore, contaminated specimens, cultures were ordered to be repeated. Identification of isolated pathogens was done using biochemical testing (Murray et al., 1995). All procedures will be considered as recommended by Clinical Laboratory Standard Institute (CLSI).

\section{Data analysis.}

Ms. Excel SPSS (statistical package for social science) will be used to determine the prevalence of common pathogens associated with UTI and coinfection

Measurements of the variable. 
The study involves the prevalence of Urinary tract infection and pathogen-associated with Urinary tract infection (dependent). The causes of infection were measured with signs and symptoms, a factor that influences sexuality and diagnosis of the disease. The risk factors of urinary tract infection were measured with prevention and management.

\section{Safety Quality Control.}

Inoculate quarter plates of the medium with a five-hour broth culture for each control organism. Use a straight wire to inoculate and a wire loop to spread the inoculum. Incubate aerobically, carbon dioxide enriched atmosphere and anaerobically at $35-37^{\circ} \mathrm{C}$ for 24 hours. Examine the degree of growth, morphology, and other characteristics of microbial colonies. Record the result of each control species and compare it to the standard reading.

\section{Ethical consideration.}

The study approval was obtained from Medical Director through the Medical Laboratory director/in charge of Kam Medical Consult Clinic. The study was also granted ethical clearance from the University of Kisubi ethical review committee. All patients' data, informed consent and bacterial isolates gathered in the study will be handled confidentially by the researcher. Further acceptable protocols of handling patient data will be adhered to. Also, laboratory coding will be used to identify patients from whom the data will be obtained.

\section{Results}

\section{Characteristics of sample collected at Kam Medical Consult Clinic.}

A total of 120 fresh urine samples were collected from patients by standard mid-stream catch method in sterile universal bottles. Among the sampled population, $33 \%$ of males had urinary tract infection while $67 \%$ of females had urinary tract infection.

Demographic information of patients presenting with UTI at Kam Medical Consult Clinic.

In regards to the sex of patients, the male were 40 while the females were 80 making the total of 120 patients. The age of patients who presented with urinary tract infection and whom the urine was collected was in the age group of 1-10 years,11- 20 years, 21 - 30 years, 31- 40 years, 41-50years,51-60 years, 61-70 years, $71-80$ years and $81-90$ years.

In the age group 1-10 years, only 4 males and 3 female had UTI, 11-20 years 2 males and females had UTI, 21- 30 years 17 males and 40 female had UTI, 31-40 years only 7 males and 7 females, 41 50 years 8 males and 3 females had UTI, $51-60$ year only 1 male and 5 female had UTI,61 - 70 year 4 males and 6 females had UTI, 71 - 80 years only I male and 1 female had UTI,81- 90 years, no male had UTI and only 3 females had UTI. Therefore the age group that presented with most UTI cases was between 21 - 30 years ( 17 males and 45 females) and the least age with UTI cases was between 71 80 years ( 1 male and 1 female). Most females with UTI cases were between the age group 21 - 30 years (45 years) whereas most males were between 21- 30 years ( 17 males ) (Table 4.1 ).

Medical status of the patients presenting with UTI at Kam Medical Consult Clinic.

The medical clinic had both in-patients and outpatients attending for the UTI service offered. Among the population sampled, the majority, $88.5 \%$ were outpatients while $11.5 \%$ were inpatients. Patients who had UTI were mainly outpatient in this medical clinic showing a significant difference in the medical status of the children.

A significant difference in the patient's UTI status to the gender of the patients was also noted. More female patients (63.3\%) had urinary tract infections (UTI) than their male counterparts (36.7\%).

\section{UTIs among children at Kam Medical Consult clinic.}

The results showed that children sampled within the age group (1-10) were found with UTI at a prevalence of $5.8 \%$. Among the 7 children enrolled, 4 males and 3 females had UTI. Therefore, this showed a significant difference in the prevalence of children in both boys and girls.

Identification of bacterial pathogens isolated from patients presenting UTI in Kam Medical Consult Clinic.

The overall prevalence of UTI among patients was $63.3 \%$ in females and $36.7 \%$ in males. A total of 120 bacterial pathogens were isolated and identified (table1). Out of the 120 bacterial pathogens isolated from the samples, 76(63.7\%) were from females samples enrolled and 44 (36.7\%) were isolated from male samples. From the 120 isolates, 92 were gram-negative, 17 gram positive, and 11 candida species. Escherichia coli 35 (29.2\%) was the highest bacterial pathogen followed by Klebsiella spp 26 (21.7), staphylococcus aureus 17 (18.3\%), unidentified coliform 13(10.8\%), Enterococcus species 11(9.2\%), Candida species 11(9.2\%), E.coli 
Table 1. 4.1: Urinary tract infection status among the age groups that presented at Kam Medical Consult Clinic.

\begin{tabular}{llll}
\hline \multicolumn{2}{l}{ Sex of patients } & & \\
Age group & Male & Female & Total \\
$1-10$ & 4 & 3 & 7 \\
$11-20$ & 2 & 8 & 10 \\
$21-30$ & 17 & 40 & 57 \\
$31-40$ & 7 & 7 & 14 \\
$41-50$ & 8 & 3 & 11 \\
$51-60$ & 1 & 5 & 6 \\
$61-70$ & 4 & 6 & 10 \\
$71-80$ & 1 & 1 & 2 \\
$81-90$ & 0 & 3 & 3 \\
Total & 44 & 76 & 120 \\
\hline
\end{tabular}

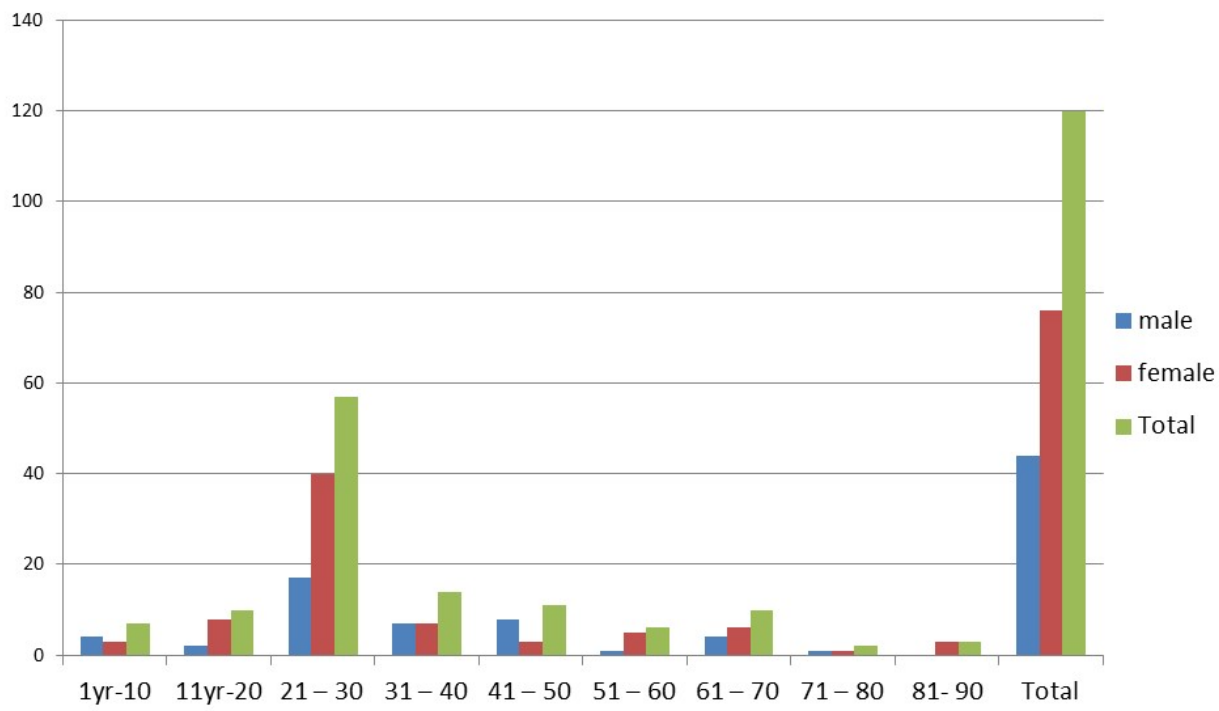

Chart 1. 4.1. The number of male and females affected with UTI to the percentage (\%)

coliform 5 (4.2\%) and pseudomonas 2(1.6\%) table 1.

\section{Discussion, Conclusion and Recommendation \\ 7 Discussion:}

The majority of patients recruited and presented with UTI in this study were females. This could be due to the tendency of clinicians sending females with the symptom of UTI to the laboratory-based merely on the observation that females are predisposed to UTIs than males. It can also be at- tributed to fact that more females presented with UTI than males. The high prevalence of infection in females (63.3\%) reported in this study is related to anatomic and pathogenic factors, for example, the short length of the urethra hence the lesser distance of bacteria ascending to the urinary tract (Aiyegero et al., 2007). This high prevalence of UTI in female in females is comparable to the prevalence rate of $18 \%$ reported in Kenya (Hannah et al., 2011). However, this study is in agreement with other reports which stress that UTI is more frequent in females than in males (Stamm; 2001, Nicolle; 2008 Salvatore, 2010). 
Table 2. acterial pathogens isolated $\mathbf{N}=120$

\begin{tabular}{lll}
\hline Bacterial isolate & Total number of isolates & Percentage of isolates (\%) \\
Escherichia coli & 35 & 29.2 \\
E.coli +coliform & 5 & 4.2 \\
Unidentified coliform & 13 & 10.8 \\
Enterococcus species & 11 & 9.2 \\
Klebsiella spp & 26 & 21.7 \\
Staphylococcus aureus & 17 & 14.2 \\
Candida species & 11 & 9.2 \\
Pseudomonas aeruginosa & 2 & 1.6 \\
Total & 120 & $100 \%$ \\
\hline
\end{tabular}

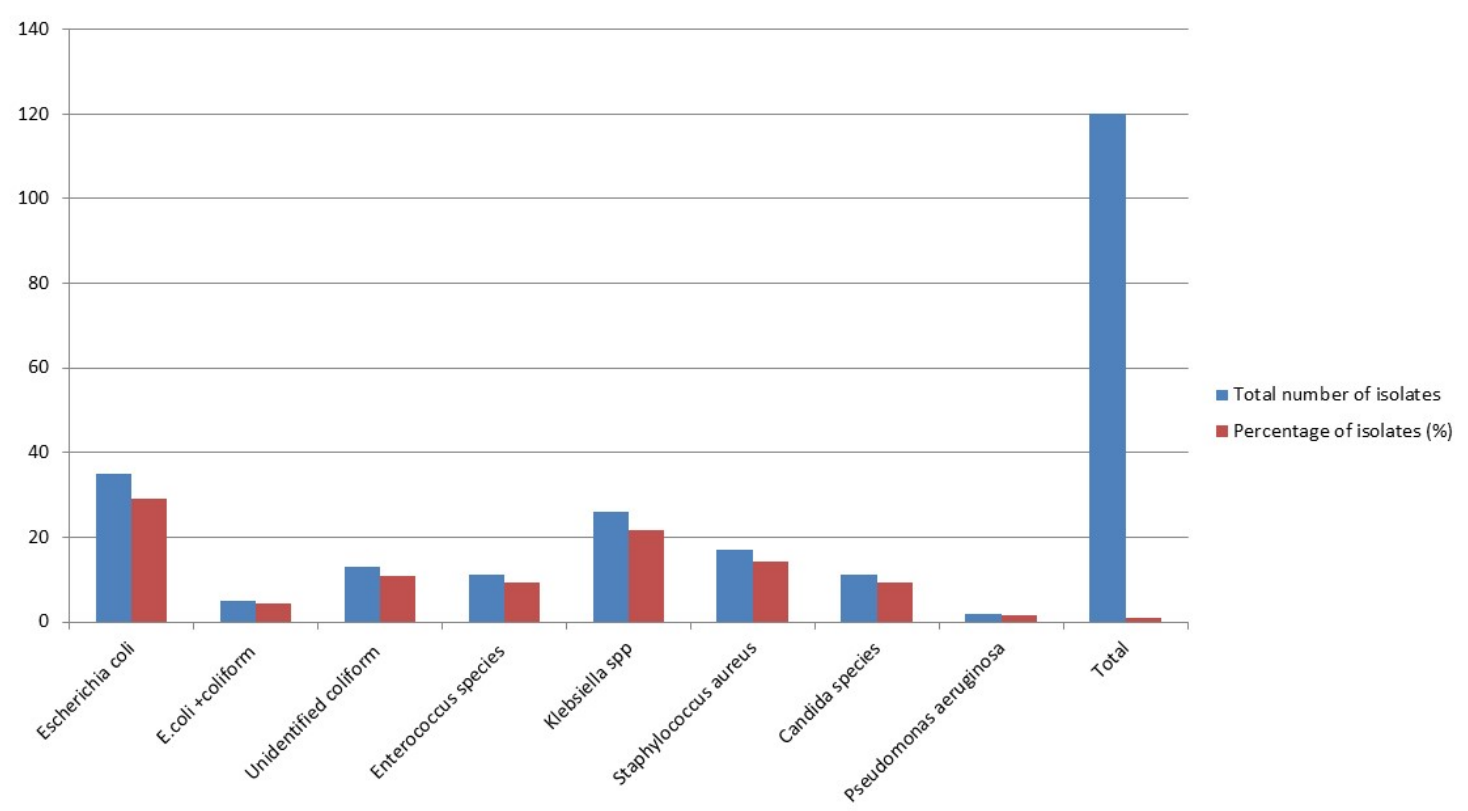

Chart 2. 4.2. Identification of 8 bacterial pathogen isolated to its percentage (\%).

The study prevalence of $63.3 \%$ in female and 36.7 in males, this correlates with range obtained in a study by Ethiopia that showed the prevalence of UTI's among patients to be between 10.5 to $39.5 \%$ (Coyne et al., 2012). The highest bacterial pathogen in this study was Escherichia coli 35 (29.2\%). This agrees with $47.6 \%$ in Nigeria and $51.4 \%$ in South Africa (Wanyama et al., 2003). However, the finding of this study is lower than (49.5\%) reported in Nigeria and South Africa (Bercion, et al., 2007). The second pathogen was Klebsiella species 26(21.7\%). This report is comparable to findings reported in Kenya at 24.0\% (Aiyegoro et al., 2007) and Nigeria 18\% (Hannah et al., 2011). In general, the present study confirmed that almost no difference exists in the type and frequency of bacteria isolated in patients. The Gram-negative bacteria isolated were more prevalent (76.7\%) than Gram-positive bacteria isolates (14.2\%) and Candida species isolates (9.2\%). This study report is similar to the study done in Jos University Teaching hospital, Nigeria that reported Gram-negative 70\% and Gram-positive 30\% isolation (Hannah et al., 2011). This could be due to the presence of a unique structure in Gramnegative bacteria which helps for attachment to the uroepithelial cells and prevent bacteria from urinary washing.

Gram-negative Enterobacteriaceae led by E.coli and Klebsiella species dominated the bacterial pathogen seen in the study, similarly to the results of other studies elsewhere (Grupta et al., 2001; Wanyama et al., 2003; Gallali et al., 2006.). S.aureus 
was the gram-positive bacteria isolated and made up only $14.2 \%$. Candida Albicans was also isolated and made up to $9.2 \%$ of the infection. The rate of candiduria found in this study is of concern, considering that the previous studies in Israel and Italy found rates ranging from 0.14- $0.77 \%$ (Parveen et al., 2011).

This study showed a significant difference in the medical clinic status. The outpatient's males and females mainly presented with UTI; this could be attributed to predisposing factors that like congestion in the working area, contaminated toilet facilities of working area, educational institutions, homestead places, and poor drainage (Hannah et al., 2011).

In this study, of 120 patients from whom urine samples were taken, 40 males (33.3\%) and 80 females (66.7\%) had UTI. This is possible because patients presented with UTI are liable indicators of infection in both males and female patients above $11-20$ years of age. This is similar to figures reported in previous studies by Aiyegoro et al., (2007) among female patients in Yola (67.2\%) in Nigeria. This incidence is however high than the prevalence rate of $18 \%$ reported by Hannah et al., (2011) in male patients hospitalized at the Kenyatta National Hospital.

\section{Conclusions.}

The finding of this study revealed that the infecting organism was the commensals of the perianal and vaginal regions. This calls for the increase in personal hygiene Enterobacteriaciae particularly E.coli is an important etiological agent causing UTIs in male and female patients presenting UTI at Kam Medical Consult Clinic. Other etiological agents identified in this study include

Klebsiella, Coliform, Enterococcus species, staphylococcus aureus, pseudomonas species, and candida species. Urine culture assists in identifying bacteria causing UTIs therefore based on culture may lead to resolution of acute symptoms and eradication of the pathogens.

The overall prevalence rate of $33.3 \%$ males and $66.7 \%$ females with urinary tract infection in this studied population, and the predominant bacterial pathogens are Escherichia coli as the leading cause of UTI in this age group. E.coli is the highest pathogen associated with urinary tract infection in both males and female patients with $34 \%$, followed by Klebsiella species with $21.7 \%$. Females are more susceptible to urinary tract infections than males. This prevalence provides great concern, as not only do UTIs pose a threat to health, but they also impose an economic and social burden due to the stigma associated with these infections.

\section{Recommendations}

Based on the results of this study, the following can be recommended.

1. There is a need to monitor the profile of etiological bacteria of UTI through culture and sensitivity regularly.

2. It is recommended that the treatment of UTI at Kam Medical Consult Clinic should be based on available evident data for example cultural methods to achieve effective therapy for patients. This will also minimize recurrent UTI and utilize the resources available.

3. Health workers should mobilize patients to ensure that they have duly checked up to identify the problem and get solved.

4. Several future research gaps worth filling are recommended. There is a need to establish the cause of recurrent UTI, and risk factors involved in infection with resistant bacterial strains.

5. Patients should not assume their automatic need for antibiotics before laboratory investigation, for example, culture and sensitivity, and should not routinely ask for antibiotics when they sick and this will greatly reduce recurrent UTI.

\section{Limitation of the study.}

Due to resource limitation, the study did not include antibiotic susceptibility assessment proper treatment and few interested patients would incur small costs, were able to perform drug susceptibility tests on a few of the specimens.

The culture growth media used was only able to culture bacteria as the causative agents of urinary tract infection thus did not assess other organisms like viruses, fungi, mycoplasma, and chlamydia trichomonas, this was because of the cost and time required. After screening for UTI, some of the patients were not available for results although the benefits of the study were explained and they were encouraged to give their contacts for feedback.

Despite taking all due precautions, and instructing women how to take clean catch urine specimen, the possibility of contamination cannot be eliminated. 


\section{List of Abbreviation and Acronyms}

ASBA -Asymptomatic bacteriuria

CDC-Centre of disease control

CFU -Colony-forming unit

CLED -Cysteine lactose electrolyte deficient Agar

KMCC- Kam medical consult clinic

RPM -Revolutions per minute

STI -Sexual transmitted infection

UNIK- University of Kisubi.

UTI -Urinary tract infection

WHO -World Health Organization.

\section{References:}

1) Bercion $R$, Gaudeuille,A.Mapouka PA,Quetahan,Y.Behounde,T (2007). Surgical site infection survet in orthopaedic surgery department of the "Hôpital communautaire de Bangui . central african Republic,bul soc pathol exot, 100.

2) Coyne,K.S; Kuagz, AM;Ireland,I;Milsom Z.S , Kopp C.R chapple (2012). Urinary incontinence and its relationship to mental health and health-related quality of life in men and women in Sweden, the United Kingdom, and the United States. Eur Urol. , 61. https://doi.org/10.1016/j.eururo.2011.07.049

3) Monica, Cheesbrough. ( 2000). District Laboratory Practice in Tropical Countries.Examination of urine and antimicrobial sensitivity testing; pp. Part 2 (c) Monica Cheesbrough.,105-143.

4) Mshana SE, Kamugisha E,.Mirabo M,Chakraborty T, Lyamuya EF, (2009). Prevalence of multiresistant gram-negative organisms in a tertiary hospital in Mwanza, Tanzania. BMC Research Notes. , 49. https://doi.org/10.1186/17560500-2-49

5) Nicolle LE (2008). uncomplicated urinary tract infection in adult and uncomplicated pyelonephritis. uro clin North Am, 35. https://doi.org/10.1016/j. ucl.2007.09.004

6) Nicolle LE, Bradly S,Colgan R,Rice JC,Schaeffer A.,Hooton TM (2005). Infectious Diseases Society of America guidelines for the diagnosis and treatment of asymptomatic bacteriuria in adults. . Clin Infect Dis, 76. https://doi.org/10.1086/427507

7) Parveen K,William DN ,Momen A.,Beguma M., (2011). Prevalence of urinary tract infection during pregnancy. J Dhaka National Med Coll Hos ., 8-12. https://doi.org/10.3329/jdnmch.v17i2.12200

8) Rahimkhani $M$, Khareri ,Daneshuar,H.,Sharifian R, ( 2008). Asymp- tomatic bacteriuria and pyuria in pregnancy. Acta Medica Iranica., 409-412.

9) Stamm W. Hooton TM (2010). The epidemiology of urinary tract infection. . Nature Reviews Urology, 60.

10) Study Guidline of Pathophisiology 5ed (2013). Elsevier Health Sciences. ISBN: chicago.

11) Whalley P. (2015). Bacteriuria of pregnancy . Am J Obstet Gynecol 97(5):, 723-738. https://doi.or g/10.1016/0002-9378(67)90458-9

12) Salvatore, S; Salvatore, S, Cattoni, E, Siesto, G, Serati, M, Sorice, P, Torella, M June 2011. (2011). urinary tract in women. european journal of obstetric and gynecology, 156. https://doi.org/10.1016/j.ejog rb.2011.01.028

13) Martin Odoki, Adamu Almustapha Aliero, Julius Tibyangye, Josephat Nyabayo Maniga, Eddie Wampande, Charles Drago Kato, Ezera Agwu, Joel Bazira, "Prevalence of Bacterial Urinary Tract Infections and Associated Factors among Patients Attending Hospitals in Bushenyi District, Uganda", International Journal of Microbiology, vol. 2019, Article ID 4246780, 8 pages, 2019. https://doi.org/10. $1155 / 2019 / 4246780$

14) Aiyegoro O.A., Igbinosa O.O., Ogunmwonyi I.N., Odjadjare E.E., IgbinosaO.E., Okoh A.I.(2007). Incidence of urinary tract infections amongchildren and adolescents in Ile-Ife, Nigeria.African Journal of Microbiology12: 13-19

15) HannahK.I., Gunturu R., Joyce M., Theresa O. (2011). The incidence of nosocomial Urinary Tract Infections in Kenyatta National Hospital.Baraton Interdisciplinary Research Journal2: 12-21.

16) Minardi, D., d'Anzeo, G., Cantoro, D., Conti, A., \& Muzzonigro, G. (2011). Urinary tract infections in women: etiology and treatment options. International journal of general medicine, 4, 333-343. http s://doi.org/10.2147/IJGM.S11767

17) CDC. April 17, 2. (2015). urinary tract infection. Retrieved 9 February 2016, 23.

18) Byamugisha, J, Andabati ,G.and Wanyama J. (March 2003). Prevalence, bacteriology and microbial sensitivity patterns among pregnant women with clinically diagnosed urinary tract infections in Mulago Hospital Labour Ward. Makerere University; M.Med dissertation of Wanyama., 143. 